\title{
MULTIPLE TIME SCALES AND CANARDS IN A CHEMICAL OSCILLATOR
}

\author{
ALEXANDRA MILIK* AND PETER SZMOLYAN †
}

\begin{abstract}
We present a geometric singular perturbation analysis of a chemical oscillator. Although the studied three-dimensional model is rather simple its dynamics are quite complex. In the original scaling the problem has a folded critical manifold which additionally becomes tangent to the fast fibers in a region relevant to the dynamics. Thus normal hyperbolicity of the critical manifold is lost in two regions. The dynamics depends crucially on effects due to the loss of normal hyperbolicity. In particular canard solutions play an essential role. We outline how rescalings and blow-up techniques can be used to prove the existence of canards in this problem and to explain other qualitative aspects of the dynamics.
\end{abstract}

Key words. Geometric singular perturbation theory, chemical oscillator, canard solutions, blow-up.

AMS(MOS) subject classifications. Primary 34A36, 58F30, 34E15.

1. Introduction. Chemically reacting systems are prominent examples of dynamical systems evolving on often widely different time scales. They do this in a way which pushes the borders of the available mathematical techniques for the analysis of slow-fast systems. We demonstrate in this paper that even in rather simple equations a sophisticated singular perturbation analysis is needed to understand the dynamics. We do this in the context of a specific system the so called 3-dimensional autocatalator. However, the phenomena and also the techniques used in the analysis are of a more general nature.

The rate equations for the 3-dimensional autocatalator have the following dimensionless form [17]

$$
\begin{aligned}
\frac{d a}{d t} & =\mu\left(\frac{5}{2}+c\right)-a b^{2}-a \\
\varepsilon \frac{d b}{d t} & =a b^{2}+a-b \\
\frac{d c}{d t} & =b-c .
\end{aligned}
$$

The variables $a, b$, and $c$ represent concentrations of three different chemical species depending on time $t$. Hence, only nonnegative values of $a, b$, and $c$ are considered in the following. The parameter $\varepsilon$ is small. The

*Institut für Angewandte und Numerische Mathematik, Technische Universität Wien, Vienna, Austria / IMA, University of Minnesota, milik@ima.umn.edu, research supported by the Austrian Science Foundation under grant J 1449-MAT.

$\dagger$ Institut für Angewandte und Numerische Mathematik, Technische Universität Wien, Vienna, Austria, ps@umbriel.tuwien.ac.at, research supported by the Austrian Science Foundation under grant Y 42-MAT 
parameter $\mu$, which will be close to $2 / 7$ throughout this paper, is considered as a bifurcation parameter.

We decided to work with the constants $5 / 2$ and $2 / 7$ to remain close to the parameter values used in the detailed numerical study [17]. Depending on $\mu$ this model shows a rich variety of dynamical behaviour known collectively as mixed mode oscillations. Mixed-mode oscillations are solutions containing certain combinations of alternating large and small amplitude excursions. See Figure 1 which shows a typical time series with one large and two small peaks. This time series is obtained by a simulation of model (1.1). As $\mu$ varies in a tiny intervall close to $2 / 7$ a Farey sequence of bifurcations of periodic orbits (of mixed mode type) leading from small Hopf-type periodic solutions to large relaxation type periodic solutions is observed. The periodic behaviour is intertwined with apparently chaotic behaviour [17]. In fact, our motivation for the study of this model was to find a geometric mechanism which explains the quite common phenomenon of mixed-mode oscillations [14].

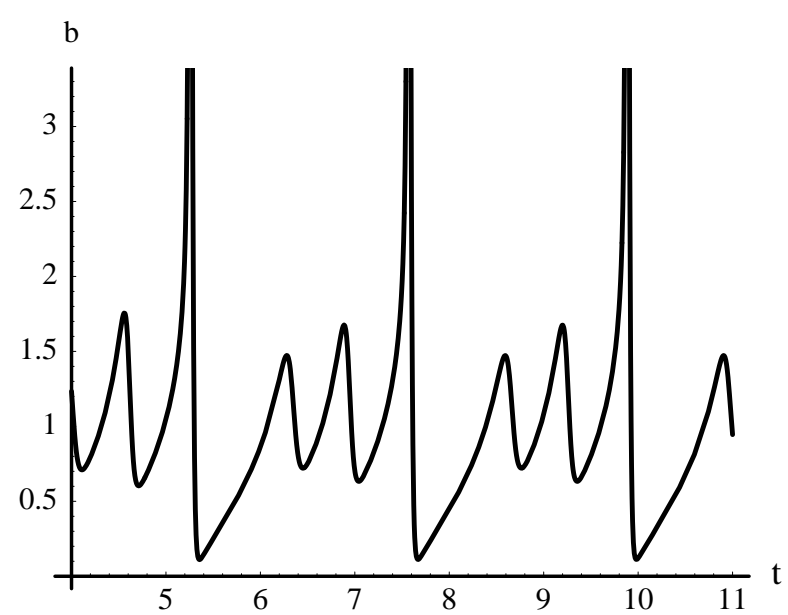

FIG. 1. Time series of the variable $b$ of the 3d-autocatalator. Parameters: $\mu=$ $0.299, \varepsilon=0.013$

System (1.1) is already in a form which suggests the application of singular perturbation theory. We follow the geometric approach to singular perturbation theory, see N. Fenichel's original paper [9] or the recent survey by $\mathrm{C}$. Jones [11]. By setting $\varepsilon=0$ we obtain the equation $a b^{2}+a-b=0$ which defines the 2-dimensional critical manifold, which is a folded surface with one unbounded branch, see Figure 2. The lower branch of the critical 
manifold is attracting, the upper branch is repelling. Away from the fold and for bounded values of $b$ the critical manifold perturbs smoothly to two slow manifolds for small $\varepsilon$. The slow manifold close to the lower branch of the critical manifold is still strongly attracting, the other one is still strongly repelling.

But recall that Fenichel's theory requires normal hyperbolicity of the critical manifold. Near the fold line and for $b \rightarrow \infty$ normal hyperbolicity of the critical manifold is lost. Thus the standard theory does not apply in these regions. Typically, a fold in a critical manifold leads to jump points, that is the orbit follows the attracting slow manifold until it reaches the fold and then jumps away almost vertically following the dynamics of the layer problem. This is the the well-known mechanism which leads to relaxation oscillations, as e.g. in Van der Pol's equation, see [16]. Thus, one could expect to see solutions of system (1.1) following the attracting slow manifold until they come close to the fold, where an "explosive" growth of $b$ would take place.

However, this behaviour is observed only at certain parts of the fold. To get an impression what else is observed and how the dynamics is organized by the slow manifold we superimposed a numerically obtained solution onto a picture of the critical manifold in phase space (see Figure 2). We summarize the results which can be obtained by such simulations:

1. As one expects, all solutions starting between the attracting and the repelling slow manifold are quickly attracted to the attracting slow manifold. The flow on the attracting slow manifold is towards the fold.

2. There exist small loops where solutions first follow the attracting branch of the slow manifold, then the repelling branch for a while until they fall back to the attracting branch.

3. There exist large loops where solutions first follow the attracting branch, then the repelling branch for a while, then jump nonvertically away from the slow manifold, make a large excursion, and eventually fall back to the attracting branch of the slow manifold.

4. As a solution comes close to the fold the value of $c$ seems to determine which behaviour occurs, i.e. there seems to exist a $c^{*}(\varepsilon) \approx 1$ such that for $c<c^{*}(\varepsilon)$ small loops occur, and for $c>c^{*}(\varepsilon)$ large loops occur. The transition between small and large loops is rather sharp.

5. After a small loop there is a net increase in the value of the variable $c$, while after a large loop there is a net decrease.

6. For solutions on the attracting slow manifold passing the fold at $c$ distinctly larger than $c^{*}(\varepsilon)$ the typical transition to an almost vertical motion occurs. For solutions on the repelling slow manifold passing the fold (in backwards time!) at $c$ distinctly smaller than $c^{*}(\varepsilon)$ the typical transition to an almost vertical motion occurs.

7. Observation 6 . is actually only correct as long as $b=O(1)$, on the 
scale $b=O(1 / \varepsilon)$ the fast dynamics is approximately along planes $a+b / \varepsilon=$ const. .

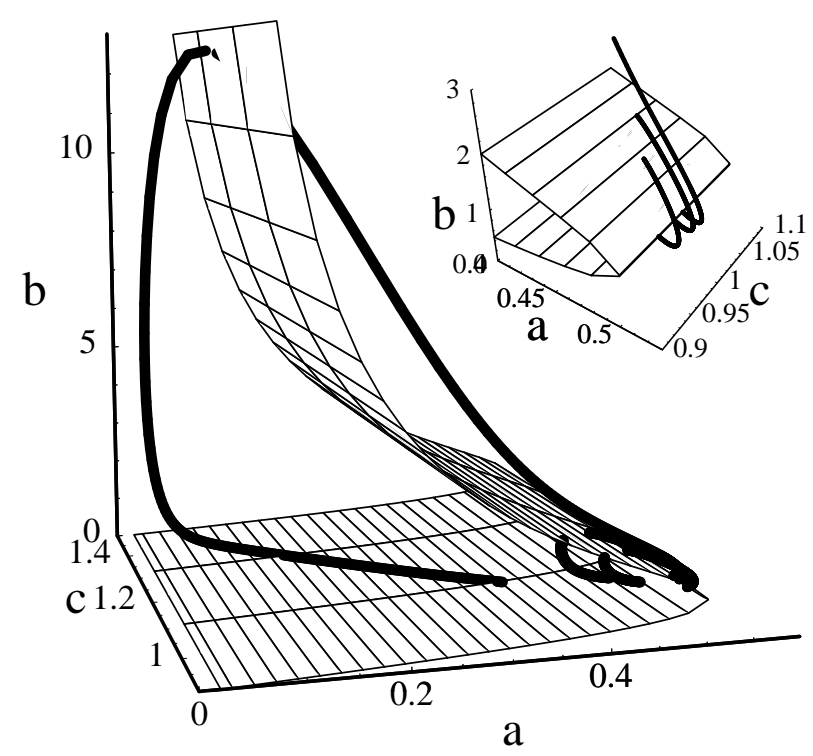

FIG. 2. Critical manifold $S$ with lower attracting branch $S_{a}$ and upper repelling branch $S_{r}$ and a typical solution of the 3-dimensional autocatalator. The parameters are $\varepsilon=0.013$ and $\mu=0.299$.

Much of this can be seen in Figure 2. It can be explained based on our main result:

Proposition 1.1. The forward in time continuation of the attracting slow manifold and the backward in time continuation of the repelling slow manifold intersect transversally in an orbit $\gamma_{\varepsilon}$. The orbit $\gamma_{\varepsilon}$ intersects the plane $b=1$ at a point $\left(a^{*}(\varepsilon), 1, c^{*}(\varepsilon)\right)$. The intersection of the slow manifolds occurs in such a way that on the left side of $\gamma_{\varepsilon}$, i.e. on the side corresponding to smaller values of $c$, the continuation of the slow attracting manifold lies below the repelling slow manifold and vice versa on the right side.

See Figure 3 for a schematic view of the intersection of the slow manifolds.

At the point $\left(a^{*}(\varepsilon), 1, c^{*}(\varepsilon)\right)$ the orbit $\gamma_{\varepsilon}$ "turns around", i.e. there the variable $a$ has its maximum along the orbit. We have introduced this point to have a precise description of the location of the orbit $\gamma_{\varepsilon}$ close to 


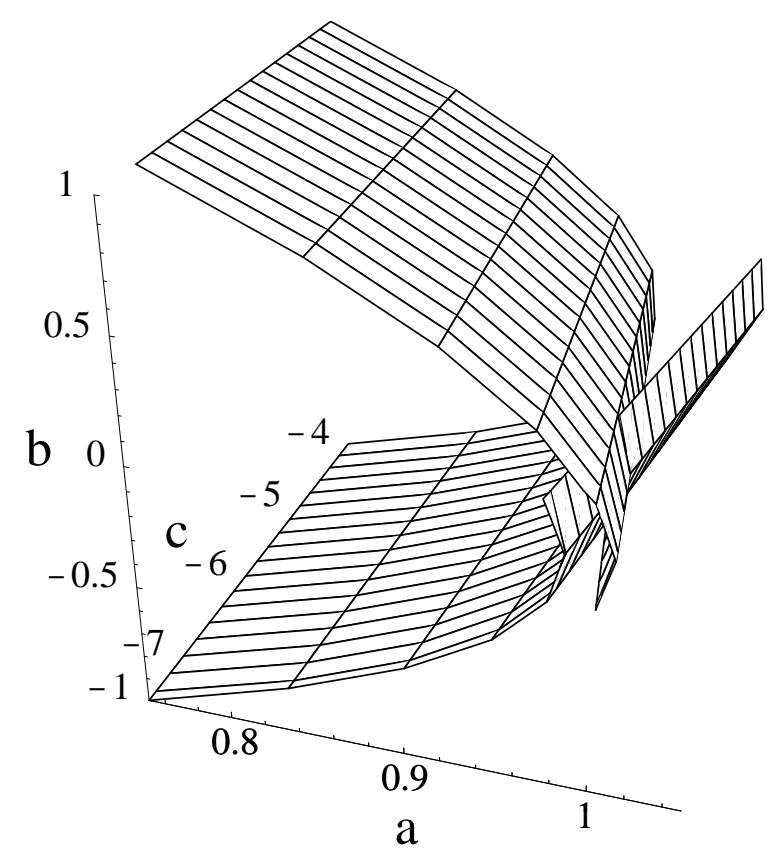

FIG. 3. Schematic view of the intersection of the perturbed slow manifolds $S_{a}^{\varepsilon}$ (lower surface) and $S_{r}^{\varepsilon}$ (upper surface).

the fold line. Clearly, this configuration of the slow manifolds explains the observations made above concerning the dynamics close to the fold. In the above description and also in the proposition we have suppressed the smooth dependence of $\gamma_{\varepsilon}$ and $c^{*}(\varepsilon)$ on the parameter $\mu$.

In singularly perturbed systems an orbit like $\gamma_{\varepsilon}$ is called a (maximal) canard. More generally orbits which follow a repelling slow manifold for an $O(1)$ amount of time are called canards $[4,6]$. Canards were discovered and first studied by means of nonstandard analysis in the Van der Poloscillator [4]. Canards arise in one-parameter families of planar singularly perturbed systems if an equilibrium passes through the fold point as the parameter varies. For a treatment of this situation which also leads to the so called singular Hopf bifurcation by matched asymptotic expansions we refer to $[8,2,5,15]$. Recently, F. Dumortier and R. Roussarie [7] gave a detailed geometric explanation and proof of the canard phenomenon in 
two dimensions by using blow-up of singularities and foliations by center manifolds as main techniques. For more details on their work we also refer to their article in this volume. We have worked out a complete blow-up analysis for the 2-dimensional autocatalator

$$
\begin{aligned}
\frac{d a}{d t} & =\mu-a b^{2}-a \\
\varepsilon \frac{d b}{d t} & =a b^{2}+a-b
\end{aligned}
$$

where the reactant corresponding to the variable $c$ is fed into the reactor at a constant rate controlled by the parameter $\mu$. Even in this much simpler problem the blow-up analysis of a planar canard (for $\mu$ close to 1) must be combined with another blow-up analysis, which is necessary to analyze the unbounded branch of the critical manifold, see [18].

Results on canards in higher dimensional systems by means of nonstandard analysis have been obtained by Benoit [3] and by means of matched asymptotic expansions in [15]. In three-dimensional problems with a folded critical manifold certain types of canards, which can be classified according the dynamics of the reduced flow on the critical manifold, occur generically. See also the survey in [1]. We will briefly comment on these types of canards in the next section.

In system (1.1) we have an even more degenerate case since there is a fixed point of the system close to the fold. In section 2 we will classify this case as a folded saddle-node. In [14] we argued analytically and numerically that mixed-mode oscillations can be explained convincingly by relating the dynamics of system (1.1) to the dynamics of a bimodal one-dimensional map. Reduction by one dimension is possible by introducing a suitable two-dimensional return map. The reduction to a one-dimensional map can be justified (somewhat heuristically) by the strong contraction of the flow near the attracting slow manifold. In the definition of the return map the canard associated with the folded saddle node plays the crucial role of separating the small loops from the large loops.

In this paper we discuss how certain parts of the construction in [14] can be put on a rigorous basis. This requires extensions of the by now well established methods of geometric singular perturbation theory to parts of the critical manifold where normal hyperbolicity breaks down. Inspired by the pioneering work of F. Dumortier and R. Roussarie [7] on the geometric analysis of canard cycles in Van der Pol's equation such a theory for higherdimensional problems is beeing developed in our group right now.

For an introduction we refer to the paper by M. Krupa and the second author in this volume. A systematic treatment of three-dimensional systems with a folded critical manifold is given in [19].

The essential tool is to blow-up degenerate equilibria corresponding to non-hyperbolic points of critical manifolds. Since the singular perturbation parameter $\varepsilon$ plays an essential role in the blow-up a planar problem leads 
to a three-dimensional problem after the blow-up. For a three-dimensional problem the blown-up problem is four-dimensional. It can be even necessary to include parameters of the problem in the blow-up. Thus the complexity of the geometry and hence of the analysis increases quickly with increasing dimensions. As we will see several different blow-ups have to be used for system (1.1).

In the 3-dimensional autocatalator the (slow) variable $c$ acts similar as the parameter $\mu$ in (1.2), however, due to the coupling of all variables a more complicated situation arises. In this paper we will restrict attention to some of the phenomena, which have to be dealt with, in a complete blow-up analysis of system (1.1). Thus, this paper can be partly seen as a report on work in progress, and also as an attempt to build a bridge from a more conventional asymptotic analysis to a geometric analysis based on blow-up methods.

We now outline the content of the rest of the paper. In Section 2 we study the reduced problem and the layer problem corresponding to system (1.1). We identify a degenerate singularity on the fold line as a folded saddle node. We show that there exists a unique solution of the reduced problem on the critical manifold which passes at the folded saddlenode from the attracting branch of the critical manifold to the repelling branch. We will prove that this solution is the singular limit of the canard.

All variables and even the singular perturbation parameter $\varepsilon$ have to be rescaled in the regions where normal hyperbolicity is lost. Actually one needs to perform a blow-up which is more than just a rescaling. However, the essential global dynamics, i.e. connections between different slow manifolds can be studied in a chart which corresponds to a rescaling of the variables. In Section 3 we introduce a rescaling suitable to study the existence of the canard. This leads to a singular perturbation of an integrable system, which can be roughly described as the normal form of a planar canard where the control parameter is replaced by another slow variable. The unperturbed integrable system has one special solution which is asymptotic to both branches of the slow manifold. We show that this solution persists under perturbation. In the original coordinates this solution corresponds to the maximal canard $\gamma_{\varepsilon}$ along which the continuations of the attracting and repelling slow manifolds intersect (see Figure 3).

In section 4 we show that the unboundedness of the critical manifold for $a \rightarrow 0+$ and the unboundedness of orbits of the layer problem require another rescaling which transforms system (1.1) into a different singularly perturbed system. Although this system is not in standard form it can be analysed by a straightforward application of geometric singular perturbation methods [9]. This leads to a mechanism which describes the large amplitude excursions. However, rigorous matching with solutions desribed in the original scaling requires also the blow-up method. 
2. Reduced dynamics and slow manifolds. In the following we study system (1.1) for $\mu$ close to $2 / 7$, which is the parameter range where mixed mode oscillations occur.

System (1.1) is a singularly perturbed problem on the slow time scale $t$ with $\varepsilon$ being the small perturbation parameter. The variable $b$ is the fast variable while $a$ and $c$ are slow variables. Taking the limit $\varepsilon \rightarrow 0$ yields the reduced problem

$$
\begin{aligned}
\frac{d a}{d t} & =\mu\left(\frac{5}{2}+c\right)-a b^{2}-a \\
0 & =a b^{2}+a-b \\
\frac{d c}{d t} & =(b-c) .
\end{aligned}
$$

This is a two-dimensional dynamical system on the critical manifold $S$ defined by the equation $a b^{2}+a-b=0$. Solving for

$$
a=\frac{b}{b^{2}+1}
$$

shows that this manifold is a folded surface in $(a, b, c)$-space with the fold line defined by $a=1 / 2, b=1$. We denote the lower, i.e. $b<1$, part of $S$ by $S_{a}$ and the upper part of $S$ by $S_{r}$ (see Figure 2).

Transforming system (1.1) to the fast timescale $\tau:=t / \varepsilon$ yields the equivalent fast system

$$
\begin{aligned}
& \frac{d a}{d \tau}=\varepsilon\left(\mu\left(\frac{5}{2}+c\right)-a b^{2}-a\right) \\
& \frac{d b}{d \tau}=a b^{2}+a-b \\
& \frac{d c}{d \tau}=\varepsilon(b-c) .
\end{aligned}
$$

By taking the limit $\varepsilon \rightarrow 0$ in this system we obtain the layer problem

$$
\begin{aligned}
& \frac{d a}{d \tau}=0 \\
& \frac{d b}{d \tau}=a b^{2}+a-b \\
& \frac{d c}{d \tau}=0 .
\end{aligned}
$$

The layer problem is a one-dimensional dynamical system in the fast variables $b$ with the slow variables $a$ and $c$ acting as parameters. The local stability of points in $S$ as steady states of the layer problem (2.6) is determined by the derivative

$$
\left.\frac{\partial\left(a b^{2}+a-b\right)}{\partial b}\right|_{S}=2 a b-\left.1\right|_{S} .
$$


Thus, points in $S_{a}$ are attracting and points in $S_{r}$ are repelling. We say that $S_{a}$ is an attracting critical manifold and $S_{r}$ a repelling critical manifold. On the fold line the derivative (2.7) is zero. $S_{a}$ is normally hyperbolic for $0 \leq a \leq a_{1}<1 / 2$, close to the fold line normal hyperbolicity is lost. Furthermore, we observe that the repelling critical manifold $S_{r}$ becomes more and more aligned with the (vertical) unstable direction as $a \rightarrow 0+$. Hence $S_{r}$ is normally hyperbolic only for $0 \leq a_{0} \leq a \leq a_{1}<1 / 2$. We conclude from Fenichel's theorems that normally hyperbolic parts of $S_{a}$ and $S_{r}$ perturb smoothly to locally invariant slow manifolds $S_{a}^{\varepsilon}$ and $S_{r}^{\varepsilon}$ of the same stability type as $S_{a}$ and $S_{r}$. In the following we will think of these manifolds as beeing extended by the forward and backward flow, however, we can not control their global dynamics by appealing to the singular limits used so far.

With a slight abuse of notation we will sometimes think of the critical manifolds as three-dimensional manifolds in $(a, b, c, \varepsilon, \mu)$ space. Note, that the slow manifolds $S_{a}^{\varepsilon}$ and $S_{r}^{\varepsilon}$ are constructed as $\varepsilon=$ const. sections of four-dimensional center-like manifolds $M_{a}$ and $M_{r}$, see [9].

Next we investigate the dynamics of the reduced problem (2.1-2.3) on the critical manifold $S$. The description of $S$ as a graph over the $(b, c)$-plane suggests to project the reduced problem into the $(b, c)$-plane. By differentiating equation (2.4) with respect to time and substituting for $d a / d t$ we obtain

$$
\begin{aligned}
\left(1-b^{2}\right) \frac{d b}{d t} & =\left(1+b^{2}\right)^{2}\left(\mu\left(\frac{5}{2}+c\right)-b\right) \\
\frac{d c}{d t} & =b-c .
\end{aligned}
$$

System (2.8) is singular at the fold, i.e. at $b=1$. The standard existence and uniqueness results for differential equations do not hold there. In particular different solutions of system (2.8) may approach the same point on the fold in finite forward or backward time. To obtain the phase portrait of this singular system we study the desingularized system

$$
\begin{aligned}
& \frac{d b}{d t}=\left(1+b^{2}\right)^{2}\left(\mu\left(\frac{5}{2}+c\right)-b\right) \\
& \frac{d c}{d t}=(b-c)\left(1-b^{2}\right),
\end{aligned}
$$

which is obtained by multiplying the right hand side of (2.8) with the term $\left(1-b^{2}\right)$. The new system has the same phase portrait as system $(2.8)$ on $S_{a}$. On $S_{r}$, that is for $b>1$, time has to be reversed in the phase portrait of system (2.9) to obtain the phase portrait of the reduced system (2.8). A computation proves:

Lemma 2.1. System (2.9) has two fixed points depending on $\mu$ :

$$
p=\left(1, \frac{1}{\mu}-\frac{5}{2}\right), \quad q=\left(\frac{5 \mu}{2(1-\mu)}, \frac{5 \mu}{2(1-\mu)}\right) .
$$


For $\mu<2 / 7$ the point $p$ is a saddle, $q$ is a stable node. For $\mu=2 / 7$ the two fixed points coalesce in the point $(1,1)$ in a transcritical bifurcation. For $\mu>2 / 7$ the point $p$ is a stable node, $q$ is a saddle.

Note that the fixed point $q$ corresponds to a genuine equilibrium of the full problem (1.1). The point $p$ on the fold line is a singular point for the reduced problem (2.8), called a folded saddle (for $\mu<2 / 7$ ) and a folded node (for $\mu>2 / 7$ ). The direction of the flow of system (2.9) has to be reversed for $b>1$ to obtain the flow of (2.8).

In this way the stable resp. unstable manifolds of the saddle become trajectories of (2.8) which pass from $S_{a}$ to $S_{r}$ resp. from $S_{r}$ to $S_{a}$ through $p$. At the folded node $p$ all trajectories pass from $S_{a}$ to $S_{r}$ along the weaker direction of its stable manifold, the only exception is one trajectory which passes tangent to the strong stable direction from $S_{a}$ to $S_{r}$. All solutions passing from $S_{a}$ to $S_{r}$ through the folded saddle or the folded node are candidates for canards.

However, we focus our attention on canards generated at the special value $\mu=2 / 7$. For this value the genuine equilibrium $q$ and the singular point $p$ coalesce in the point $(1,1)$. Thus the situation is the 3 -dimensional extension of the 2-dimensional scenario for canards, where an equilibrium passes through a fold point as a parameter variese. From this point of view the phenomena studied here in a concrete problem are clearly of a general nature.

Now we give a more detailed description of the dynamics of the reduced problem for $\mu=2 / 7$. We start by analysing the flow of system (2.9).

LEMma 2.2. The center direction for the nonhyperbolic fixed point $q=(1,1)$ of system (2.9) with $\mu=2 / 7$ is the vector $(2,7)$. There exists a center manifold on which the flow is weakly contracting towards $q$ for $b<1$, i.e. in $S_{a}$, and weakly repelling for $b>1$, i.e. in $S_{r}$. There exists a strong stable manifold tangent to the vector $(1,0)$.

Proof. The linearization at the equilibrium is the matrix

$$
\left(\begin{array}{cc}
-4 & \frac{8}{7} \\
0 & 0
\end{array}\right)
$$

which implies all assertions except the description of the center flow. The assertion about the center-flow follows from a center manifold computation.

To obtain the phase portrait of the reduced problem (2.1-2.3) shown in Figure 4 one has to reverse the direction of the flow on $S_{r}$. We call the singular point $(1,1)$ a folded saddle-node. We remark that we use this terminology because of the phase portrait of problem (2.9) for fixed $\mu=2 / 7$. As we have seen in Lemma 2.1 the unfolding of this degenerate singularity by $\mu$ is a transcritical bifurcation of equilibria for system (2.9). The folded saddle node is a singular point of the reduced problem. Solutions on the former center manifold converge to the folded saddle-node with an exponential rate as $t \rightarrow \infty$. This follows because of a cancelation between 
the quadratic zero of the flow of system (2.9) on the center manifold and the factor $(1-b)$ of the derivative $d b / d t$ in system $(2.8)$.

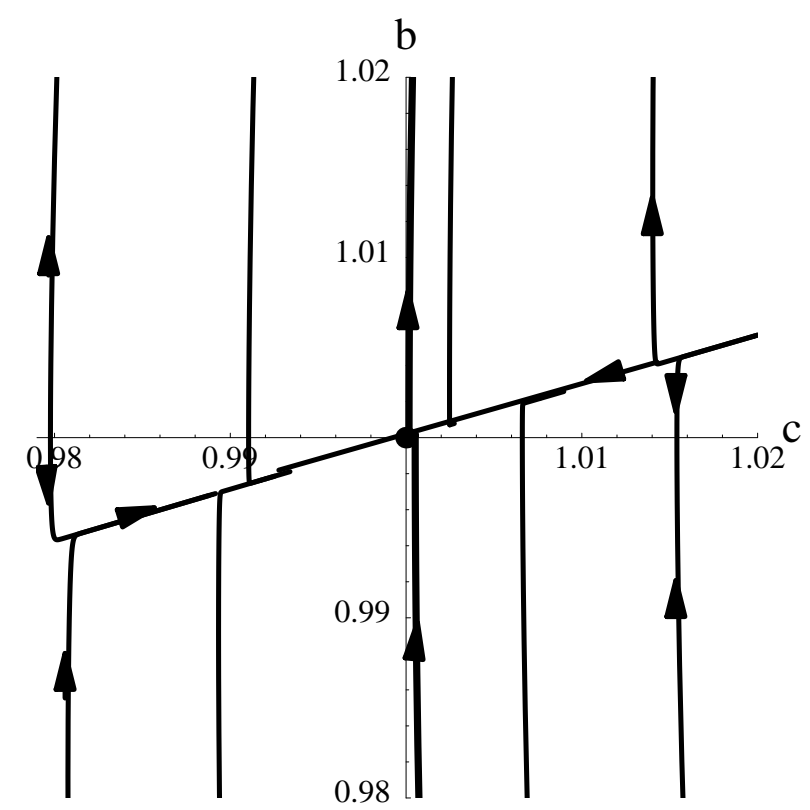

FIG. 4. Computed phase portrait of the reduced problem in the (c,b)-plane with folded saddle-node $q$ (black point) and singular canard $\gamma$ (bold line).

The strong stable manifold becomes a solution $\gamma$ which crosses the fold exactly at the folded saddle node. Moreover, $\gamma$ is the only solution with this property. We call the orbit $\gamma$ the singular canard. Trajectories on $S_{a}$ on the left side of $\gamma$ are attracted by the former center manifold. All solutions starting in $S_{a}$ on the right side of $\gamma$ approach points on the fold in finite time. The folded saddle node is the only point on the critical manifold $S$ where a transition from $S_{a}$ to $S_{r}$ is possible. At points on the fold with $c>1$ the reduced flow can not be continued in forward time, that is a transition to a fast motion must occur. Similarly, at points on the fold with $c<1$ the reduced flow can not be continued backwards, that is a transition to a fast motion must occur in backwards time.

In the next section we analyse the behaviour of the slow manifolds for $\mu$ close to $2 / 7$ for small positive $\varepsilon$. In particluar we will show that the singular canard persists as a maximal canard $\gamma_{\varepsilon}$ along which the extended 
slow manifolds $S_{a}^{\varepsilon}$ and $S_{r}^{\varepsilon}$ intersect.

3. Dynamics close to the folded saddle-node. The starting point of the analysis close to the folded saddle-node for small $\varepsilon$ and $\mu$ close to $2 / 7$ are equations (2.5)

$$
\begin{aligned}
& \frac{d a}{d \tau}=\varepsilon\left(\mu\left(\frac{5}{2}+c\right)-a b^{2}-a\right) \\
& \frac{d b}{d \tau}=a b^{2}+a-b \\
& \frac{d c}{d \tau}=\varepsilon(b-c) \\
& \frac{d \varepsilon}{d \tau}=0 \\
& \frac{d \mu}{d \tau}=0
\end{aligned}
$$

which are the equations on the fast scale extended by the trivial equations for the parameters $\varepsilon$ and $\mu$. All points on the fold line are equilibria of system (3.1) for $\varepsilon=0$. The linearization of system (3.1) at a point $(1 / 2,1, c, 0, \mu)$ on the fold line has $\lambda=0$ as an eigenvalue of multiplicity five. At other points of the critical manifold $S$, which are also equilibria for $\varepsilon=0$, the linearization has one nonzero (negative in $S_{r}$, positive in $S_{a}$ ) eigenvalue and an eigenvalue $\lambda=0$ of multiplicity four. The degenerate equilibria on the fold must be treated with the blow-up method.

3.1. Blow-up and charts. In the following we focus on the folded saddle-node $(1 / 2,1,1,0,2 / 7)$. We first perform a coordinate transformation which shifts the folded saddle-node to the origin and blow up the shifted variables according to the transformation

$$
\begin{aligned}
a & =\frac{1}{2}+\bar{r}^{2} \bar{a} \\
b & =1+\bar{r} \bar{b} \\
c & =1+\bar{r} \bar{c} \\
\varepsilon & =\bar{r}^{2} \bar{\varepsilon} \\
\mu & =\frac{2}{7}+\bar{r} \bar{\mu} .
\end{aligned}
$$

Note that the weights, i.e. the powers of $\bar{r}$, used for the individual variables are the same as in the analysis of planar canards at a fold point. The variable $c$ and the parameter $\mu$ have both weight one, they both play a role analogous to the parameter in the case of planar canards.

We define the manifold $B=S^{4} \times \mathbb{R}$ and $B_{0}=S^{4} \times\left[0, r_{0}\right)$ for sufficiently small $r_{0}>0$ and regard the transformation (3.2) as the mapping

$$
\phi: B_{0} \rightarrow \mathbb{R}^{5}
$$


with $(\bar{a}, \bar{b}, \bar{c}, \bar{\varepsilon}, \bar{\mu}) \in S^{4}$ defined by $\bar{a}^{2}+\bar{b}^{2}+\bar{c}^{2}+\bar{\varepsilon}^{2}+\bar{\mu}^{2}=1$. We will be mostly interested in nonnegative values of $\bar{\varepsilon}$ corresponing to nonnegative $\varepsilon$. Note, that all objects defined in the following extend smoothly to $\bar{r}<0$, hence there are no problems associated with the boundary $\bar{r}=0$ of $B_{0}$. Let $X$ denote the vectorfield coresponding to (3.1). Since $X$ vanishes at the point $(1 / 2,1,1,0,2 / 7)$ there exists a vectorfield $\bar{X}$ on $B_{0}$ such that

$$
\phi_{*} \bar{X}=X,
$$

where $\phi_{*}$ is induced by $\phi$. It remains to study the vectorfield $\bar{X}$ on the manifold $B_{0}$. In fact to analyse system (3.1) in a neighborhood of the point $(1 / 2,1,1,0,2 / 7)$ it suffices to study $\bar{X}$ on $B_{0}$ for small $r_{0}>0$.

Instead of working directly on $B$ we will use suitable charts. The most important chart $K_{1}$ is obtained by setting $\bar{\varepsilon}=1$ in transformation (3.2). The corresponding coordinates $\left(a_{1}, b_{1}, c_{1}, \mu_{1}, r_{1}\right) \in \mathbb{R}^{4} \times \mathbb{R}_{0}^{+}$cover the part of $B$ with $\bar{\varepsilon}>0$. In these coordinates the blow-up transformation has the form

$$
\begin{aligned}
a & =\frac{1}{2}+r_{1}^{2} a_{1} \\
b & =1+r_{1} b_{1} \\
c & =1+r_{1} c_{1} \\
\varepsilon & =r_{1}^{2} \\
\mu & =\frac{2}{7}+r_{1} \mu_{1} .
\end{aligned}
$$

As $\left(a_{1}, b_{1}, c_{1}, \mu_{1}\right)$ tends to infinity the corresponding point in $S^{4}$ tends to a point $(\bar{a}, \bar{b}, \bar{c}, 0, \bar{\mu})$ on the "equator" of $S^{4}$. Hence, the dynamics at "infinity" in the chart $K_{1}$ is more conveniently studied in other charts. Actually, we will need just one other chart $K_{2}$ which is obtained analogously by setting $\bar{a}=-1$ in the blow-up transformation (3.2). The corresponding coordinates $\left(b_{2}, c_{2}, \varepsilon_{2}, \mu_{2}, r_{2}\right) \in \mathbb{R}^{4} \times \mathbb{R}_{0}^{+}$cover the part of $B$ with $\bar{a}<0$. In these coordinates the blow-up transformation has the form

$$
\begin{aligned}
a & =\frac{1}{2}-r_{2}^{2} \\
b & =1+r_{2} b_{2} \\
c & =1+r_{2} c_{2} \\
\varepsilon & =r_{2}^{2} \varepsilon_{2} \\
\mu & =\frac{2}{7}+r_{2} \mu_{2} .
\end{aligned}
$$

The change of coordinates between $K_{1}$ and $K_{2}$ in their overlap domain $a_{1}<0$ resp. $\varepsilon_{2}>0$ is described in the following table. 


$$
\begin{array}{c|c}
K_{1} \text { to } K_{2} & K_{2} \text { to } K_{1} \\
\hline b_{2}=b_{1}\left(-a_{1}\right)^{-1 / 2} & a_{1}=-\varepsilon_{2}^{-1} \\
c_{2}=c_{1}\left(-a_{1}\right)^{-1 / 2} & b_{1}=b_{2} \varepsilon_{2}^{-1 / 2} \\
\varepsilon_{2}=-a_{1}^{-1} & c_{1}=c_{2} \varepsilon_{2}^{-1 / 2} \\
\mu_{2}=\mu_{1}\left(-a_{1}\right)^{-1 / 2} & \mu_{1}=\mu_{2} \varepsilon_{2}^{-1 / 2} \\
r_{2}=r_{1}\left(-a_{1}\right)^{1 / 2} & r_{1}=r_{2} \varepsilon_{2}^{1 / 2}
\end{array}
$$

We introduce the following notation: $\bar{P}$ denotes an object in the blowup which corresponds to object $P$ in the original problem. If $\bar{P}$ is described in one of the charts then $P_{1}$ denotes the object in chart $K_{1}$ and similarly $P_{2}$ is the same object written in chart $K_{2}$. This convention is used only when necessary, mostly to distinguish between various invariant manifolds.

3.2. Study in $K_{1}$, family rescaling. The vectorfield $\bar{X}$ written in chart $K_{1}$ is obtained by inserting transformation (3.4) into system (3.1). A computation gives the equations

$$
\begin{aligned}
a_{1}^{\prime} & =-b_{1}+\frac{2}{7} c_{1}+\frac{7}{2} \mu_{1}+r_{1}\left(-2 a_{1}-\frac{1}{2} b_{1}^{2}+\mu_{1} c_{1}\right)+O\left(r_{1}^{2}\right) \\
b_{1}^{\prime} & =2 a_{1}+\frac{1}{2} b_{1}^{2}+2 r_{1} a_{1} b_{1}+O\left(r_{1}^{2}\right) \\
c_{1}^{\prime} & =r_{1}\left(b_{1}-c_{1}\right) \\
\mu_{1}^{\prime} & =0 \\
r_{1}^{\prime} & =0
\end{aligned}
$$

where superscript prime denotes differentiation with respect to the rescaled time $\tau_{1}=r_{1} \tau$, that is we have divided the right-hand side of the equation by $r_{1}$. In the chart $K_{1}$ the system is still a two-parameter family of threedimensional vectorfields, the blow-up transformation in these coordinates is just a rescaling of variables and parameters.

It is interesting to observe that system (3.6) is again a singularly perturbed problem, however, with fast variables $a_{1}, b_{1}$ and the slow variable $c_{1}$. The singular perturbation parameter is $r_{1}$. A computation gives

Lemma 3.1. The singularly perturbed system (3.6) has a one-dimensional critical manifold

$$
S_{1}=\left\{\left(-\frac{1}{4}\left(\frac{2}{7} c_{1}+\frac{7}{2} \mu_{1}\right)^{2}, \frac{2}{7} c_{1}+\frac{7}{2} \mu_{1}, c_{1}\right): c_{1} \in \mathbb{R}\right\} .
$$

The branch of $S_{1}$ corresponding to $c_{1}<-49 \mu_{1} / 4$ is attracting, the branch of $S_{1}$ corresponding to $c_{1}>-49 \mu_{1} / 4$ is repelling. At $c_{1}=-49 \mu_{1} / 4$ a (degenerate) Hopf bifurcation occurs. The reduced flow on $S_{1}$ is governed 
by the equation

$$
\dot{c}_{1}=-\frac{5}{7} c_{1}+\frac{7}{2} \mu_{1}
$$

where the derivative is with respect to $t_{1}:=r_{1} \tau_{1}$.

The flow on $S_{1}$ has an attracting equilibrium point at $c_{1}=49 \mu_{1} / 10$. A computation shows that this equilibrium, written in the original variables, corresponds to the equilibrium $q$ described in Lemma 2.1. We expect that a similar relation exists between the critical manifold $S_{1}$ and the center manifold described in Lemma 2.2. We have not checked this in detail.

For $\mu_{1} \neq 0$ the reduced flow on the critical manifold $S_{1}$ passes through the Hopf point, for $\mu_{1}=0$ the equilibrium on the critical manifold and the Hopf point coalesce at $c_{1}=0$. This situation is reminiscent of the delayed Hopf bifurcation, see the paper by A. Neishtadt in this volume and references therein. Thus, a more detailed analysis of the local dynamics seems to be difficult. F. Dumortier has informed us that related problems are discussed in his paper with $\mathrm{R}$. Roussarie in this volume. These connections will be investigated in future work. Fortunately, an understanding of these phenomena is not needed to prove the existence of the canard solution, since as we will see the canard solution stays away from $S_{1}$. However, it can very well be that these phenomena play a role in a complete understanding of mixed-mode oscillations.

The canard solution is obtained as a perturbation of a special solution of system (3.6) with $r_{1}=0$. This gives the equations

$$
\begin{aligned}
& a_{1}^{\prime}=-b_{1}+\frac{2}{7} c_{1}+\frac{7}{2} \mu_{1} \\
& b_{1}^{\prime}=2 a_{1}+\frac{1}{2} b_{1}^{2},
\end{aligned}
$$

where $c_{1}$ acts as a paramter in the same way as $\mu_{1}$. For $2 / 7 c_{1}+7 / 2 \mu_{1}=0$ we obtain equation

$$
\begin{aligned}
& a_{1}^{\prime}=-b_{1} \\
& b_{1}^{\prime}=2 a_{1}+\frac{1}{2} b_{1}^{2},
\end{aligned}
$$

well known from the analysis of planar canards $[7,5,2,8]$. The function

$$
H\left(a_{1}, b_{1}\right)=\left(2-2 a_{1}-\frac{b_{1}^{2}}{2}\right) e^{a_{1}}
$$

is a constant of motion. The level curves of $H$ are shown in Figure 5. The origin is a center surrounded by periodic orbits. The bold line is the special orbit $\gamma_{1}$ corresponding to $H=0$. A parametrization of $\gamma_{1}$ is given by $\left\{\left(-\tau_{1}^{2}+1,2 \tau_{1}\right): \tau_{1} \in \mathbb{R}\right\}$. Note that for $a_{1} \ll 0$ the lower branch of $\gamma_{1}$ is strongly attracting, while the upper branch is strongly repelling. We 
will see that this behaviour is inherited from the stability properties of the critical manifolds $S_{a}$ and $S_{r}$. In a suitable compactification of the plane the orbit $\gamma_{1}$ is a heteroclinic orbit connecting two equilibria. The nature of these "equilibria at infinity" will become clear in the following subsection.

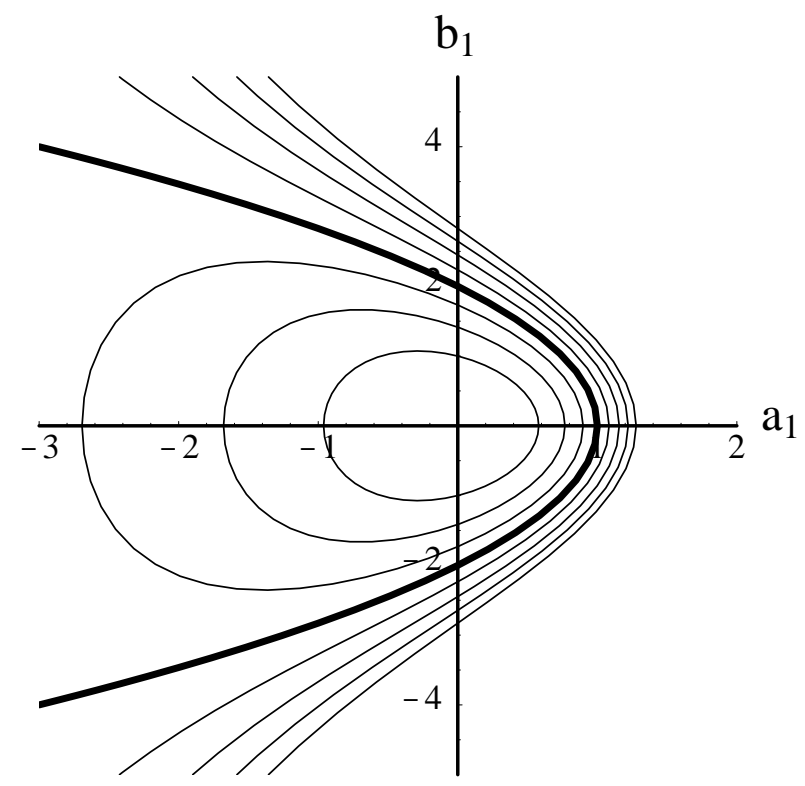

FIG. 5. Levelcurves and the special orbit $\gamma_{1}$ (boldcurve). Inside of this orbit there are periodic orbits surrounding a center at the origin, outside there is a family of unbounded curves. of system (3.8).

Lemma 3.2. As $2 / 7 c_{1}+7 / 2 \mu_{1}$ passes through zero the orbit $\gamma_{1}$ of system (3.7) considered as a heteroclinic orbit connecting two "equilibria at infinity" breaks transversally.

This crucial property of system (3.7) is already known from the analysis of planar canards in [7]. A proof of the Lemma based on a variant of the Melnikov method is given in Section 3.4. However, some care is needed to justify the Melnikov calculation, in particular we need a better understanding of system (3.6) at infinity. We will show that there exist four-dimensional invariant manifolds $M_{a, 1}$ resp. $M_{r, 1}$ of system (3.6), which are strongly attracting resp. repelling for $a_{1} \ll 0$. We will show that these manifolds can be constructed as center-manifolds of equilibria of $\bar{X}$ on the "equator" of the sphere $S^{4}$. Recall that the sphere $S^{4}$ is the blown- 
up locus of the degenerate point on the fold line which we are studying. The necessary computations are done in chart $K_{2}$.

3.3. Study in $K_{2}$, phase-directional rescaling. The vectorfield $\bar{X}$ in the chart $K_{2}$ is obtained by inserting transformation (3.5) into system (3.1). A computation gives the equations

$$
\begin{aligned}
b_{2}^{\prime} & =-2+\frac{1}{2} b_{2}^{2}+\frac{1}{2} \varepsilon_{2} b_{2}\left(-b_{2}+\frac{2}{7} c_{2}+\frac{7}{2} \mu_{2}+O\left(r_{2}\right)\right) \\
c_{2}^{\prime} & =\frac{1}{2} \varepsilon_{2} c_{2}\left(-b_{2}+\frac{2}{7} c_{2}+\frac{7}{2} \mu_{2}+O\left(r_{2}\right)\right) \\
\varepsilon_{2}^{\prime} & =\varepsilon_{2}^{2}\left(-b_{2}+\frac{2}{7} c_{2}+\frac{7}{2} \mu_{2}+O\left(r_{2}\right)\right) \\
\mu_{2}^{\prime} & =\frac{1}{2} \varepsilon_{2} \mu_{2}\left(-b_{2}+\frac{2}{7} c_{2}+\frac{7}{2} \mu_{2}+O\left(r_{2}\right)\right) \\
r_{2}^{\prime} & =-\frac{1}{2} \varepsilon_{2} r_{2}\left(-b_{2}+\frac{2}{7} c_{2}+\frac{7}{2} \mu_{2}+O\left(r_{2}\right)\right),
\end{aligned}
$$

where superscript prime denotes differentiation with respect to the rescaled time $\tau_{2}=r_{2} \tau$, i.e. we have divided the right-hand side of the equation by $r_{2}$. In the chart $K_{2}$ we loose the property that the original problem is a twoparameter family of three-dimensional vectorfields. The two parameters are now treated as dynamical variables. Note however, that the equations for $\varepsilon_{2}$ and $\mu_{2}$ are obtained by differentiating the relations $\varepsilon=r_{2} \varepsilon_{2}$ and $\mu=2 / 7+r_{2} \mu_{2}$, and using $d \varepsilon / d \tau=0, d \mu / d \tau=0$. This implies that $r_{2} \varepsilon_{2}$ and $r_{2} \mu_{2}$ are two constants of motion.

System (3.10) has four invariant hyperplanes defined by $\varepsilon_{2}=0, r_{2}=0$, $c_{2}=0$, and $\mu_{2}=0$. In the plane $\varepsilon_{2}=0$ the equations become very simple

$$
\begin{aligned}
b_{2}^{\prime} & =-2+\frac{1}{2} b_{2}^{2}+O\left(r_{2}\right) \\
c_{2}^{\prime} & =0 \\
\mu_{2}^{\prime} & =0 \\
r_{2}^{\prime} & =0 .
\end{aligned}
$$

Hence, there exist functions $h_{a}\left(r_{2}\right)=-2+O\left(r_{2}\right)$ and $h_{r}\left(r_{2}\right)=2+O\left(r_{2}\right)$ both defined for $[0, \rho)$ for some $\rho>0$ such that the equations $\varepsilon_{2}=0$ and $b_{2}=h_{a}\left(r_{2}\right)$ resp. $b_{2}=h_{r}\left(r_{2}\right)$ define three-dimensional manifolds $S_{a, 2}$ resp. $S_{r, 2}$ of equilbria of system (3.10). These manifolds are nothing but the branches $S_{a}$ and $S_{r}$ of the critical manifold (trivially extended in $\mu$ direction) of the layer problem (2.6). However, due to the blow-up we have gained normal hyperbolicity of these manifolds at $r_{2}=0$. The relevant eigenvalue is $\lambda= \pm 2$ at points $\left( \pm 2, c_{2}, \mu_{2}, 0\right)$.

In the plane $r_{2}=0$ the governing equations are

$$
b_{2}^{\prime}=-2+\frac{1}{2} b_{2}^{2}+\frac{1}{2} \varepsilon_{2} b_{2}\left(-b_{2}+\frac{2}{7} c_{2}+\frac{7}{2} \mu_{2}\right)
$$




$$
\begin{aligned}
c_{2}^{\prime} & =\frac{1}{2} \varepsilon_{2} c_{2}\left(-b_{2}+\frac{2}{7} c_{2}+\frac{7}{2} \mu_{2}\right) \\
\varepsilon_{2}^{\prime} & =\varepsilon_{2}^{2}\left(-b_{2}+\frac{2}{7} c_{2}+\frac{7}{2} \mu_{2}\right) \\
\mu_{2}^{\prime} & =\frac{1}{2} \varepsilon_{2} \mu_{2}\left(-b_{2}+\frac{2}{7} c_{2}+\frac{7}{2} \mu_{2}\right) .
\end{aligned}
$$

All points $\left( \pm 2, c_{2}, 0, \mu_{2}\right)$ are equilibria with one hyperbolic eigenvalue $\lambda=$ \pm 2 and a zero eigenvalue of multiplicity three.

We restrict our attention to a neighborhood of the equilibrium points $p_{a}=(-2,0,0,0,0)$ and $p_{r}=(2,0,0,0,0)$ since these are needed in the analysis of the canards. In the following theorem we denote the canonical basis of $\mathbb{R}^{5}$ by $e_{i}, i=1, \ldots, 5$.

THEOREM 3.1. System (3.10) has the following properties:

1. There exists an attracting four-dimensional center-manifold $M_{a, 2}$ at $p_{a}$ with a stable foliation. The strong stable manifold at $p_{a}$ is tangent to $e_{1}$. The center-manifold $M_{a, 2}$ is tangent to span $\left\{-e_{1}+\right.$ $\left.e_{3}, e_{2}, e_{4}, e_{5}\right\}$ at $p_{a}$.

2. There exists a repelling four-dimensional center-manifold $M_{r, 2}$ at $p_{r}$ with an unstable foliation. The strong unstable manifold at $p_{r}$ is tangent to $e_{1}$. The center-manifold $M_{r, 2}$ is tangent to span $\left\{e_{1}+\right.$ $\left.e_{3}, e_{2}, e_{4}, e_{5}\right\}$ at $p_{r}$.

3. The intersection of $M_{a, 2}$ with the invariant space defined by $r_{2}=$ $c_{2}=\mu_{2}=0, \varepsilon_{2}>0$ is a single solution $\gamma_{a, 2}$ of system (3.10) which converges to $p_{a}$ tangent to $-e_{1}+e_{3}$ as $\tau_{2} \rightarrow-\infty$. The solution $\gamma_{a, 2}$ is the part of the special solution $\gamma_{1}$ of system (3.8) corresponding to $\tau_{1} \in(-\infty, 0]$ rewritten in the chart $K_{2}$.

4. The intersection of $M_{r, 2}$ with the invariant space defined by $r_{2}=$ $c_{2}=\mu_{2}=0, \varepsilon_{2}>0$ is a single solution $\gamma_{r, 2}$ of system (3.10) which converges to $p_{r}$ tangent to $e_{1}+e_{3}$ as $\tau_{2} \rightarrow \infty$. The solution $\gamma_{r, 2}$ is the part of the special solution $\gamma_{1}$ of system (3.8) corresponding to $\tau_{1} \in[0, \infty)$ rewritten in the chart $K_{2}$.

Proof. The first two assertions follow from standard center-manifold theory and the above observations on the spectrum of the linearization. In the invariant space $r_{2}=c_{2}=\mu_{2}=0$ system (3.10) simplifies to

$$
\begin{aligned}
b_{2}^{\prime} & =-2+\frac{1}{2} b_{2}^{2}-\frac{1}{2} \varepsilon_{2} b_{2}^{2} \\
\varepsilon_{2}^{\prime} & =-\varepsilon_{2}^{2} b_{2} .
\end{aligned}
$$

The center manifold of this planar system at $(-2,0)$ is tangent to $(-1,1)$. Its branch corresponding to $\varepsilon_{2}>0$ is unique. A computation based on the formulas for the change of coordinates between the charts $K_{1}$ and $K_{2}$ shows, that the orbit $\gamma_{1}$ rewritten in the chart $K_{2}$ converges to $(-2,0)$ tangent to the center direction $(-1,1)$ as $\tau_{1} \rightarrow-\infty$. The uniqueness of the branch of the center manifold in $\varepsilon_{2}>0$ implies Assertion 3. Assertion 4 is proved the same way. 
Note that sections $\varepsilon=$ const. of the "blown-down" manifolds $\Phi\left(\bar{M}_{a}\right)$ and $\Phi\left(\bar{M}_{r}\right)$ are precisely the slow manifolds $S_{a}^{\varepsilon}$ and $S_{r}^{\varepsilon}$ of the original problem (1.1). The center-manifolds $M_{a, 2}$ and $M_{r, 2}$ are not unique, however all of them contain the special solution $\gamma_{1}$ written in chart $K_{2}$. Hence, our results hold for an arbitrary choice of these center-manifolds, respectively of the slow manifolds $S_{a}^{\varepsilon}$ and $S_{r}^{\varepsilon}$.

3.4. Existence of canards. Now we are ready to prove Proposition 1.1. As a preparatory step we express the center-manifolds $M_{a, 2}$ and $M_{r, 2}$ from Theorem 3.1 in chart $K_{1}$ to obtain (locally) invariant manifolds $M_{a, 1}$ and $M_{r, 1}$ of system (3.6).

THEOREM 3.2. The following assertions hold for system (3.6):

1. The forward in time continuation of $M_{a, 1}$ and the backward in time continuation of $M_{r, 1}$ intersect along the special orbit $\gamma_{1}$.

2. There exists a smooth distance function $d: U \rightarrow \mathbb{R}$, where $U$ is a small neighborhood of $(0,0,0)$ in $\left(c_{1}, \mu_{1}, r_{1}\right)$-space, such that in a tubular neighborhood of $\gamma_{1}$ the manifolds $M_{a, 1}$ and $M_{r, 1}$ intersect if and only if $d\left(c_{1}, \mu_{1}, r_{1}\right)=0$.

3. The manifolds $M_{a, 1}$ and $M_{r, 1}$ intersect transversally, the distance function satisfies

$$
\frac{\partial d}{\partial c_{1}}(0,0,0)=\frac{2}{7} \sqrt{\pi}
$$

The equation $d\left(c_{1}, \mu_{1}, r_{1}\right)=0$ can be solved for $c_{1}=c_{1}^{*}\left(\mu_{1}, r_{1}\right)$ where $c_{1}^{*}$ is a smooth function defined in a neighborhood of $(0,0)$.

Proof. The first assertion follows from Theorem 3.1 and the existence of the special orbit $\gamma_{1}$. The orbit $\gamma_{1}$ intersects the plane $b_{1}=0$ transversally, see Figure 5. We use this plane as a section and define

$$
d\left(c_{1}, \mu_{1}, r_{1}\right):=a_{1}^{-}\left(c_{1}, \mu_{1}, r_{1}\right)-a_{1}^{+}\left(c_{1}, \mu_{1}, r_{1}\right),
$$

where $a_{1}^{-}$resp. $a_{1}^{+}$are the $a_{1}$ coordinate of the points where $M_{a, 1}$ resp. $M_{r, 1}$ intersect the plane $b_{1}=0$, see Figure 6. This proves Assertion 2 . Transversality of the intersection and the precise value of $\partial d / \partial c_{1}$ follow from the Melnikov calculation given below, which also proves Lemma 3.2. Solvability of the equation $d\left(c_{1}, \mu_{1}, r_{1}\right)=0$ with respect to $c_{1}$ follows from the implicit function theorem.

The linearization of system (3.7) around $\gamma_{1}$ is

$$
\left(\begin{array}{c}
a_{1}^{\prime} \\
b_{1}^{\prime}
\end{array}\right)=\left(\begin{array}{cc}
0 & -1 \\
2 & 2 \tau_{1}
\end{array}\right)\left(\begin{array}{c}
a_{1} \\
b_{1}
\end{array}\right) .
$$

The (up to a multiplicative constant) unique bounded solution of the corresponding adjoint equation is

$$
\psi\left(\tau_{1}\right)=\left(\begin{array}{c}
e^{-\tau_{1}^{2}} \\
\tau_{1} e^{-\tau_{1}^{2}}
\end{array}\right)
$$




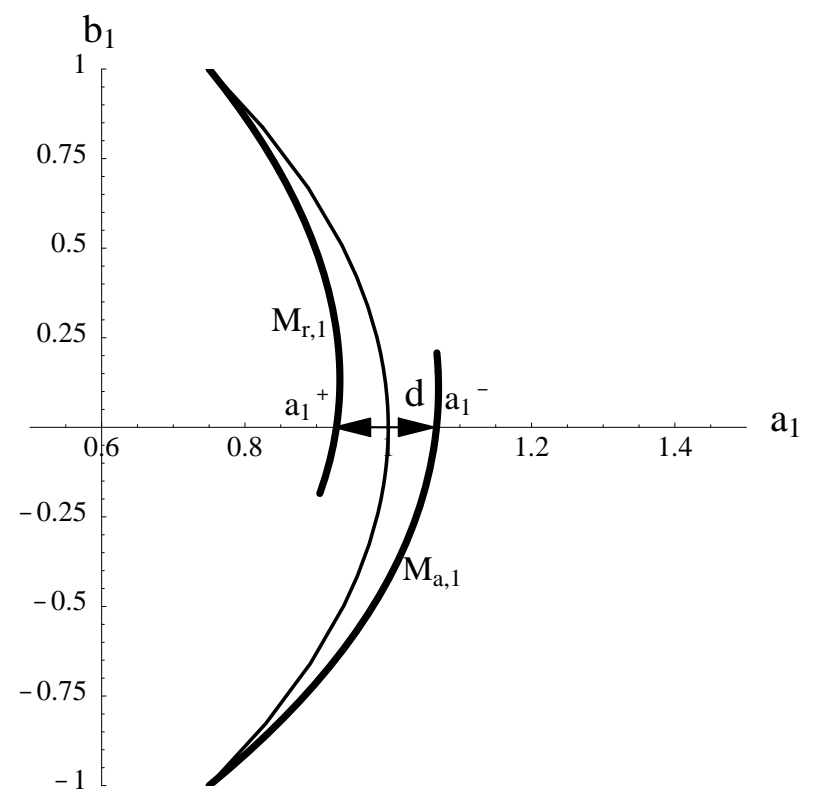

FIG. 6. The special solution $\gamma_{1}$ and the distance function $d$ for the manifolds $M_{a, 1}$ and $M_{r, 1}$ (bold curves).

Note that $\psi(0)$ is a unit vector in the direction normal to $\gamma_{1}$, that is in the direction in which the distance is measured. Let $f$ denoted the right hand side of system (3.7). It can be shown by adapting the usual derivation of the Melnikov Integral, see e.g. [10], that

$$
\frac{\partial d}{\partial c_{1}}(0,0,0)=\int_{-\infty}^{\infty} \psi\left(\tau_{1}\right) \cdot \frac{\partial f}{\partial c_{1}}\left(\gamma_{1}\left(\tau_{1}\right)\right) d \tau_{1} .
$$

A computation of the integral gives the value in Assertion 3. $\square$

We remark that the derivative $\frac{\partial d}{\partial r_{1}}(0,0,0)$ can be computed by a similar Melinikov calculation. The justification of this calculation is more subtle, see [13].

The inequality $\frac{\partial d}{\partial c_{1}}>0$ implies that the manifolds $M_{a, 1}$ and $M_{r, 1}$ intersect in the following way. In the section $b_{1}=0$ the inequality $a_{1}^{-}<$ $a_{1}^{+}$holds for $c_{1}<c_{1}^{*}\left(\mu_{1}, r_{1}\right)$, i.e. the manifold $M_{a, 1}$ lies to the "left" of $M_{r, 1}$. For $c_{1}>c_{1}^{*}\left(\mu_{1}, r_{1}\right)$ the manifold $M_{a, 1}$ lies to the "right" of $M_{r, 1}$, see Figure 3). 
The following observation relates the break up of these manifolds to the small oscillations of mixed-mode oscillations. For simplicity we fix $\mu_{1}=0$. It is not hard to see that the part of $M_{a, 1}$ corresponding to $r_{1}=0, c_{1}<0$ spirals towards the attracting part of the one-dimensional slow manifold $S_{1}$ described in Lemma 3.1. Similarly, the part of $M_{r, 1}$ corresponding to $r_{1}=0, c_{1}>0$ spirals towards the repelling part of the one-dimensional slow manifold $S_{1}$ in backwards time. This behaviour will persist for $r_{1}>0$ at least for $c_{1}$ sufficiently far away from zero. For $c_{1}$ close to zero the situation is more complicated because of the Hopf bifuractions described in Lemma 3.1. We do not attempt a more detailed analysis at this point because of the complications described in the discussion following Lemma 3.1.

Clearly, all our results about the manifolds $M_{a, 1}$ resp. $M_{r, 1}$ and $M_{a, 2}$ resp. $M_{r, 2}$, are in fact results about the existence and intersection of invariant manifolds $\bar{M}_{a}$ resp. $\bar{M}_{r}$ of the blown up vector-field $\bar{X}$ in $B_{0}$ for $r_{0}$ sufficiently small. This gives:

Proof. (of Proposition 1.1) All assertions of the proposition follow from Theorem 3.1 and Theorem 3.2 by means of transformation (3.2).

We conclude this section by summarizing the dynamics close to the fold. On the left side of the maximal canard the attracting slow manifold $S_{a}^{\varepsilon}$ lies below the repelling slow manifold $S_{r}^{\varepsilon}$, while on the right side the attracting manifold lies above the repelling manifold. Here we refer to the side of the maximal canard correponding to smaller values of the variable $c$ as the left side and to the side corresponding to larger values of $c$ as the right side. All solutions starting on the attracting manifold on the right side of the maximal canard will leave the vicinity of the fold. A solution starting on the left side of the maximal canard falls back to the attracting slow manifold (at a somewhat larger value of c) thus forming a small loop. Depending on which side of the canard the solution falls on, the solution either leaves the fold or goes through another small loop. Thus, left of the canard the attracting slow manifold scrolls up in a multilayered structure. Eventually, the solution lands on the right side of the canard and leaves the vicinity of the fold. The geometry of the repelling manifold is generated in a similar way in backwards time.

In the next section we analyse the dynamics after a solution leaves the vicinity of the fold.

4. Large relaxation oscillations. As mentioned in the introduction all orbits which leave the fold come back to the attracting manifold after a large excursion due to a global mechanism. It is clear that such a behaviour is not present in the singular limits we have studied so far. However, our approach of treating system (1.1) as singularly perturbed with slow variables $a, c$ and fast variable $b$ is only valid as long as $b=O(1)$. For $b=O(1 / \varepsilon)$ new scales arise and a different asymptotic analysis is needed. 
This is best seen if the variables are rescaled according to

$$
\begin{aligned}
a & =A \\
b & =\frac{1}{\varepsilon} B \\
c & =C \\
t & =\varepsilon^{2} T .
\end{aligned}
$$

This introduces the new time-scale $T$, which is the fastest time-scale in the problem. In the new variables system (1.1) has the form

$$
\begin{aligned}
& \frac{d A}{d T}=\varepsilon^{2} \mu\left(\frac{2}{7}+C\right)-A B^{2}-\varepsilon^{2} A \\
& \frac{d B}{d T}=A B^{2}+\varepsilon^{2} A-\varepsilon B \\
& \frac{d C}{d T}=\varepsilon B-\varepsilon^{2} C .
\end{aligned}
$$

System (4.2) is also a singularly perturbed system, however not in standard form, that is the slow variables are not constant along the fibers of the layer problem. The layer problem is obtained by setting $\varepsilon=0$

$$
\begin{aligned}
& \frac{d A}{d T}=-A B^{2} \\
& \frac{d B}{d T}=A B^{2} \\
& \frac{d C}{d T}=0 .
\end{aligned}
$$

For system (4.3) the planes $A=0$ and $B=0$ are manifolds of equilibria, that is they are critical manifolds. For $B \geq \delta_{0}, \delta_{0}>0$ the plane $A=0$ is normally hyperbolic, the relevant eigenvalue is $\lambda=-B^{2}$. The critical manifold $B=0$ is nowhere normally hyperbolic, the relevant linearization is zero. Along the line $C$-axis the problem is even more degenerate due to the intersection of the two critical manifolds.

The dynamics of the layer problem is rather simple. The two critical manifolds are connected by orbits of the layer problem. An arbitrary point $\left(A_{0}, 0, C_{0}\right)$ is connected to the point $\left(0, A_{0}, C_{0}\right)$ by a heteroclinic orbit of the layer problem in a straight line, since $A+B=$ const. . The approach to $\left(0, A_{0}, C_{0}\right)$ as $T \rightarrow \infty$ is exponential, the approach to $\left(A_{0}, 0, C_{0}\right)$ as $T \rightarrow-\infty$ is in the center direction, hence only algebraic. These straight lines are the fibers of the stable manifold of the plane $A=0$.

On the plane $A=0$ a reduced problem can be defined by projecting the vector field (4.2) onto the plane $A=0$ along the fibers of the stable manifold and deviding by $\varepsilon$, see [9]. This gives

$$
\frac{d A}{d \tau}=0
$$




$$
\begin{aligned}
& \frac{d B}{d \tau}=-B \\
& \frac{d C}{d \tau}=B,
\end{aligned}
$$

where $\tau=\varepsilon T$. Note that $\tau$ is the "slow time-scale" for system (4.2) and the "fast time-scale" for system (1.1). The reduced problem (4.4) is also very simple. A solution starting at an arbitrary point $\left(0, B_{0}, C_{0}\right)$ converges for $\tau \rightarrow \infty$ exponentially to the point $\left(0,0, B_{0}+C_{0}\right)$ along a straight line. Fenichel's theorems imply that the critical manifold $A=0$, the reduced dynamics and the stable foliation perturb smoothly for small $\varepsilon$ as long as $B \geq \delta_{0}, \delta_{0}>0$.

This gives the following singular limit description of the mechanism generating the large loops. Assume that a solution of the original system (1.1) jumps away from the critical manifold $S_{r}$ at a point $\left(a_{0}, b_{0}, c_{0}\right)$. Written in the rescaled variables (4.1) this point is $\left(a_{0}, \varepsilon b_{0}, c_{0}\right)$. In the limit $\varepsilon \rightarrow 0$ this gives the initial condition $\left(a_{0}, 0, c_{0}\right)$ for the layer problem (4.3). The point $\left(a_{0}, 0, c_{0}\right)$ is connected with the point $\left(0, a_{0}, c_{0}\right)$ by a heteroclinic orbit of the layer problem (4.3). The point $\left(0, a_{0}, c_{0}\right)$ is connected to the point $\left(0,0, a_{0}+c_{0}\right)$ by an orbit of the reduced problem (4.4).

This end point has coordinates $\left(0,0, a_{0}+c_{0}\right)$ also in the original scaling and is thus a point lying on the critical manifold $S_{a}$ of the original system (1.1). Thus, the the solution comes back to the attracting critical manifold $S_{a}$ at a point which depends on the $a$ and $c$ coordinates of the point where the solution left the critical manifold $S_{r}$. On the critical manifold $S_{a}$ the flow of the reduced system (2.2) carries the point $\left(0,0, a_{0}+c_{0}\right)$ back to the neighborhood of the fold line and the whole process starts again.

This singular limit analysis of the return mechanism cannot be made rigorous by standard arguments because of the loss of hyperbolicity near the critical manifold $B=0$. Also the matching at take-off from the critical manifold $S_{r}$ needs to be done more rigorously. As mentioned in the introduction we have worked out a rigorous analysis based on the blow-up method for system (1.2), see [18]. Work in progress indicates that the above arguments can be made rigorous by a variant of the blow-up method.

5. Conclusions and Outlook. We presented a geometrical analysis of the dynamics of the autocatalator. One of our intentions was to present this prototypical example from theoretical chemistry to a mathematicaly oriented audience. We showed that this simple looking problem contains a surprisingly large number of scales. The other intention was to demonstrate that geometric singular perturbation theory combined with the blow-up method is well suited to analyse such problems.

In addition to making our analysis more complete, there are open questions we have not even touched, yet. Fore instance, in a study of mixed mode oscillation in the BDK-model [12], which has a similar structure, the 
author proposes that the mixed-mode oscillations are related to a tangency of the stable and unstable manifolds of a periodic orbit. How does this relate to the present analysis? It seems possible to investigate this conjecture with the methods used in this paper. A related problem is to understand the origin of the apparently chaotic dynamics in problems which exhibit mixed-mode oscillations.

Acknowledgments: We would like to thank M. Krupa and M. Wechselberger for useful discussions. Part of this research was carried out while both authors were visiting the IMA. We would like to thank the IMA for support and for providing a stimulating research environment. A. M. also thanks the Geometry Center for technical support.

\section{REFERENCES}

[1] V. Arnol'D, Dynamical Systems V, vol. 5 of Encyclopedia of Mathematical Sciences, Springer Verlag, Berlin, 1994.

[2] S. BAer AND T. ERneUX, Singular hopf bifurcation to relaxation oscillations, SIAM Journal of Applied Mathematics, 46 (1986), pp. 721-739.

[3] E. Benoit, Systèmes lents-rapides dans $\mathbb{R}^{3}$ et leurs canards, Société Mathématique de France Astérisque, 109-110 (1983), pp. 159-191.

[4] E. Benoit, J.-L. Callot, F. Diener, and M. Diener, Chasse au canard, Collect. Math., 31 (1981), pp. 37-119.

[5] B. Branksma, Critical phenomena in dynamical systems of van der Pol type, Thesis, Rijksuniversiteit te Utrecht, Nederlands, 1993.

[6] M. DiEnER, The canard unchained or how fast/slow dynamical systems bifurcate, The Mathematical Intelligencer, 6 (1984), pp. 38-49.

[7] F. Dumortier and R. Roussarie, Canard cycles and center manifolds, Memoirs of the American Mathematical Society, 121 (1996).

[8] W. ECKHAUS, Relaxation oscillations including a standard chase on french ducks, vol. 985 of Springer Lecture Notes Math., Springer, 1983, pp. 449-494.

[9] N. FenicheL, Geometric singular perturbation theory, Journal of Differential Equations, 31 (1979), pp. 53-98.

[10] J. Guckenheimer And P. Holmes, Nonlinear Oscillations, Dynamical Systems, and Bifurcations of Vector Fields, Springer, New York, 1983.

[11] C. Jones, Geometric singular perturbation theory, vol. 1609 of Springer Lecture Notes Math., Springer, 1995, pp. 44-120.

[12] M. T. KOPER, Bifurcations of mixed-mode oscillations in a three-variable autonomous van der pol-duffing model with a cross-shaped phase diagram, Physica D, 80 (1995), pp. 72-94.

[13] M. KRUPa AND P. Szmolyan, Extending geometric singular perturbation theory to nonhyperbolic points, in preparation, (1998).

[14] A. Milik, P. Szmolyan, H. Löffelmann, AND E. Gröller, The geometry of mixed-mode oscillations in the 3d-autocatalator, International Journal of Bifurcation and Chaos, 8 (1998).

[15] E. Mishchenko, Y. Kolesov, A. Y. Kolesov, And N. K. Rozov, Asymptotic methods in singularly perturbed systems, Monographs in Contemporary Mathematics, Consultants Bureau, New York, A Division of Plenum Publishing Corporation 233 Spring Street, New York, N.Y. 10013, 1994.

[16] E. MishChenKo AND N. K. RozOV, Differential equations with small parameters and relaxation oscillations, Plenum Press, New York, 1980.

[17] V. Petrov, S. K. Scott, And K. Showalter, Mixed-mode oscillations in chemical systems, Journal of Chemical Physics, 97 (1992), pp. 6191-6198. 
[18] P. Szmolyan, Geometry of singular perturbations: a case study, in preparation, (1998).

[19] M. WeChSELBERGer, Singularly perturbed folds and canards in $\mathbb{R}^{3}$, Thesis, Technische Universität Wien, Austria, 1998. 